\title{
Interference microscopy under double-wavelet analysis: A new approach to studying cell dynamics
}

Sosnovtseva, Olga; Pavlov, A.N.; Brazhe, N.A.; Brazhe, A.R.; Erokhova, L.A.; Maksimov, G.V.; Mosekilde, Erik

Published in:

Physical Review Letters

Link to article, DOI:

10.1103/PhysRevLett.94.218103

Publication date:

2005

Document Version

Publisher's PDF, also known as Version of record

Link back to DTU Orbit

Citation (APA):

Sosnovtseva, O., Pavlov, A. N., Brazhe, N. A., Brazhe, A. R., Erokhova, L. A., Maksimov, G. V., \& Mosekilde, E. (2005). Interference microscopy under double-wavelet analysis: A new approach to studying cell dynamics.

Physical Review Letters, 94(21), 218103. https://doi.org/10.1103/PhysRevLett.94.218103

\section{General rights}

Copyright and moral rights for the publications made accessible in the public portal are retained by the authors and/or other copyright owners and it is a condition of accessing publications that users recognise and abide by the legal requirements associated with these rights.

- Users may download and print one copy of any publication from the public portal for the purpose of private study or research.

- You may not further distribute the material or use it for any profit-making activity or commercial gain

- You may freely distribute the URL identifying the publication in the public portal 


\title{
Interference Microscopy under Double-Wavelet Analysis: A New Approach to Studying Cell Dynamics
}

\author{
O. V. Sosnovtseva, ${ }^{1}$ A. N. Pavlov, ${ }^{2}$ N. A. Brazhe, ${ }^{3}$ A. R. Brazhe, ${ }^{3}$ L. A. Erokhova, ${ }^{3}$ G. V. Maksimov, ${ }^{3}$ and E. Mosekilde ${ }^{1}$ \\ ${ }^{1}$ Department of Physics, The Technical University of Denmark, 2800 Kongens Lyngby, Denmark \\ ${ }^{2}$ Physics Department, Saratov State University, Astrakhanskaya Street 83, Saratov, 410026, Russia \\ ${ }^{3}$ Biophysics Department, Biology Faculty, Moscow State University, Leninskie Gory, 1, Building 12, 119992, Moscow, Russia
}

(Received 27 January 2005; published 3 June 2005)

This Letter combines a novel experimental approach to the study of intracellular processes with a newly developed technique for multimode time-series analysis. Experiments are performed on isolated pond snail (Lymnaea stagnalis) neurons. Local variations in the cellular refractive index as detected by laser interference microscopy are related to the processes in the cell. A wavelet analysis shows the presence of several identifiable modes in the membrane and intracellular dynamics, and a double-wavelet analysis reveals nonlinear interactions between the regulatory processes in the form of mutual frequency and amplitude modulations.

DOI: $10.1103 /$ PhysRevLett.94.218103

PACS numbers: 87.17.-d, 05.45.Tp, 87.16.-b

Neuronal activity involves a multitude of regulatory processes that occur over many different time scales both in the plasma membrane and in the various cytoplasmic compartments. Information concerning the mutual interactions among these processes could contribute significantly to a better understanding of cellular regulation and function. However, traditional experimental techniques (such as patch clamping, intracellular registration of membrane potentials, and fluorescent microscopy) in general only allow us to study the processes individually. These techniques are also more or less invasive and, hence, may distort the processes or damage neuron structures. Besides, conventional statistical methods are not well suited for a study of nonlinear mode interaction. Hence, we need both an alternative experimental approach and a new data analysis technique to complement existing methods of cellular research. In this Letter we demonstrate how the novel technique of interference microscopy together with time-series analysis based on wavelet transform can be applied to study multimode dynamical phenomena in living cells.

Interference microscopy was originally developed to monitor surfaces of optical devices, video discs, and integral schemes with submicron resolution. The technique has also been applied to estimate the size of such biological nanoscale structures as mitochondria and viruses $[1,2]$. The technique is particularly sensitive to processes that are coordinated in time and space and this allows us to study cooperative phenomena in cells $[3,4]$.

Experimental technique.-Interference microscopy is based on the measurement of the optical path difference between the laser beam transmitted through an object and reflected from the bottom mirror layer and a reference beam reflected from the control mirror [1,2]. The measured value is normalized to the wavelength in order to obtain the so-called phase height of the object in a particular point

$$
\Phi=\frac{\phi_{0}-\phi_{\mathrm{obj}}}{2 \pi} \frac{\lambda}{2}-\Phi_{0}
$$

Here $\phi_{0}$ and $\lambda$ are the initial phase and the wavelength of the laser beam, respectively, $\phi_{\text {obj }}$ denotes the phase shift caused by the object, and $\Phi_{0}$ is a constant phase shift determined by the choice of phase reference point.

For a heterogeneous object the phase height is

$$
\Phi(x, y)=\int_{0}^{Z}\left(n_{\mathrm{obj}}(x, y, z)-n_{s}\right) d z-\Phi_{0},
$$

with $n_{s}$ being the (constant) refractive index of physiological saline and $n_{\mathrm{obj}}(x, y, z)$ the refractive index of the cell in a point of distance $z$ from the mirror. Phase height values $\Phi(x, y)$ in all points of the object form a phase height relief of the cell. Changes of the phase height due to modifications of the cell geometry and/or refractive index are mainly determined by $n_{\mathrm{obj}}(x, y, z)$. The spatial resolution of the method depends on the laser wavelength and is better for shorter wavelengths. In order to avoid photodamage, cells should be tested for the laser light absorbency. We have found that snail neurons only absorb weakly in the region of the laser light $(532 \mathrm{~nm})$ and thus their photodamage is minimal.

Experimental procedure. - Neurons were mechanically isolated from the buccal ganglia of the pond snail $L$. stagnalis and placed in a containment chamber with standard physiological saline for snails [5]. Figures 1(a) and 1(b) present an optical photography and a phase height relief of a neuron. The phase height relief reflects the location of various organelles, the cytoskeleton, and different plasma membrane structures. The highest (white) points in the phase height relief (Fig. 1) correspond to positions with the highest optical density. Systematic changes of the neuron phase height provide data on the dynamics of the local refractive index resulting from pro- 
(a)

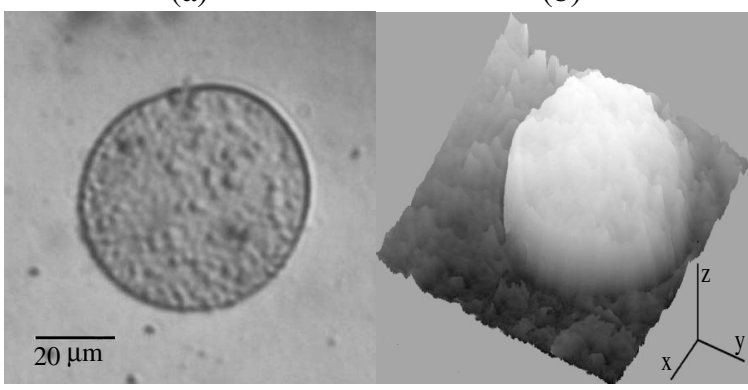

FIG. 1. (a) Optic photograph of an isolated pond snail neuron. (b) Phase height relief of the same neuron with $x$ and $y$ representing geometrical size (bar indicates $10 \mu \mathrm{m}$ ) and $z$ phase height (bar is $200 \mathrm{~nm}$ ). The phase height relief was obtained with a laser beam of wavelength $\lambda=532 \mathrm{~nm}$.

cesses in the plasma membrane and inside the cell. Data acquisition was performed by means of horizontal scans of 4,8 , or 16 points in the phase height relief. The sampling interval for individual point was 8,16 , or $32 \mathrm{~ms}$, respectively. For statistical analysis, we performed 6-10 measurements in neuron center. The recording points were uniformly distributed along the scan line (done by the microscope software). During the experiments, the overall shape and volume of the neurons remained constant. Thus, the observed changes of the phase height can be related directly to changes of the cellular refractive index.

Data analysis. - To display the endogenous modes of the cell and possible interactions between the neuronal processes we use the technique of double-wavelet analysis. The wavelet-transform of a signal $x(t)$ is obtained from $[6,7]$ :

$$
T_{x}(a, t)=\frac{1}{\sqrt{a}} \int_{-\infty}^{\infty} x(u) \psi^{*}\left(\frac{u-t}{a}\right) d u,
$$

where $\psi$ is a "mother" function that should be solitonlike with zero average. $T_{x}(a, t)$ are the wavelet coefficients and $a$ is a time-scale parameter. The details of this transform (e.g., the choice of $\psi$ ) depend on the problem to be solved. In analysis of rhythmic components, the Morlet function is typically considered in the simplified form

$$
\psi(\tau)=\pi^{-1 / 4} \exp \left(j 2 \pi f_{0} \tau\right) \exp \left[-\frac{\tau^{2}}{2}\right]
$$

The choice of $f_{0}$ allows us to search for a compromise between the localizations of the wavelet in the time and frequency domains. The relation between the scale $a$ and the central frequency for the mother function is $f=1 / a$.

Besides the coefficients $T_{x}(a, t)$, the energy density of the signal $x(t)$ in the time-scale plane can be estimated: $E_{x}(a, t)=C a^{-1}\left|T_{x}(a, t)\right|^{2}$, with $C$ being a parameter that depends on the choice of mother function. $E_{x}(a, t)$ represents a surface in three-dimensional space whose sections at fixed time moments correspond to the local energy spectrum. To simplify the visualization of this surface, the dynamics of only the local maxima of $E_{x}(a, t)$ or $E_{x}(f, t)$, i.e., the time evolution of the spectral peaks, will be considered.

In order to study interaction properties of the different modes, we propose to use the following approach. The time dependence of the instantaneous frequency of the considered mode is taken as an input signal for a second wavelet transform (1) [8]. Again, the wavelet coefficients and the energy density are estimated and the simplified visualization of the energy density is considered. The latter will contain information about all modes involved in the modulation process. In the case of nonstationary dynamics we can examine how the features (characteristics) of the frequency modulation change with time. By analogy with the instantaneous frequency, we can consider the instantaneous amplitude of the fast dynamics and, thereby, examine the amplitude modulation of the rhythmic activity.

Using recordings of refractive index versus time we obtain the power spectra shown in Fig. 2. Note that the low and high frequency bands are represented separately since different values of $f_{0}$ are used for their calculation ( $f_{0}=1$ and $f_{0}=5$, respectively) and because the involved rhythmic components have very different powers. The peaks distinguished in the spectrum are correlated to the rates of change in the refractive index during the observation time. To give biological significance to our analysis, we will try to identify each peak with a particular process within the cell.

It is known that changes of the neuronal intrinsic optical properties are caused by cellular electric activity, redistribution of intra- and extra-cellular ions, and changes of the volume, shape, and location of various organelles $[9,10]$. So far, however, the temporal properties of these processes and their mutual interdependences have remained practically unexplored. We suppose that the low frequency components $(0.1-5 \mathrm{~Hz})$ correspond to plasma membrane processes: (i) frequencies around $0.1 \mathrm{~Hz}$ may originate from complex reorganizations in the plasma membrane such as lipid rafts and protein movements, changes of the amount of membrane-bound ions and, related hereto, local fluctuations of the membrane fluidity and transmembrane potential; (ii) $\mathrm{Cd}^{2+}$ ions (a blocker for $\mathrm{Ca}^{2+}$ channels) eliminate frequencies in the range $0.2-0.4 \mathrm{~Hz}$. This illustrates the role of the voltage-dependent $\mathrm{Ca}^{2+}\left(\mathrm{Ca}_{V}^{2+}\right)$ channels at these frequencies [11]; (iii) neurons in the ganglia of
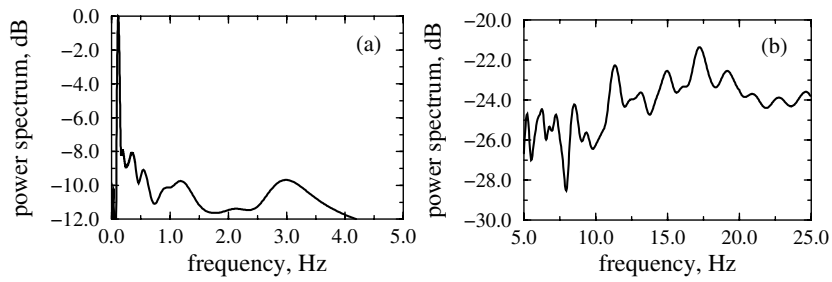

FIG. 2. Power spectra calculated via the wavelet technique for (a) the low and (b) high frequency bands, respectively. 
mollusca Helix aspersa are known to possess an intrinsic $1 \mathrm{~Hz}$ activity and a nonspecific induced activity at 1.5 and $3 \mathrm{~Hz}$ [12]. Moreover, Peixoto and Ramirez [13] found that during the first three hours after isolation, Helix neurons show a spontaneous rhythmic activity with $1-10 \mathrm{~Hz}$ frequencies. Based on this information we suggest that the prominent spectral peaks with frequencies around 1 and $3 \mathrm{~Hz}$ correspond to subthreshold changes of the membrane potential and/or to spontaneous rhythmic activity.

In the high frequency range $(10-20 \mathrm{~Hz})$ the peaks are broader and harder to distinguish than in the low frequency range. This is likely to be the result of a superposition of many membrane and cytoplasmic processes. We suppose that peaks at $10-20 \mathrm{~Hz}$ correspond to the (i) spontaneous bursting activity of neurons [14] and (ii) movements of vesicles and organelles in the cytoplasm. The last assumption is in accordance with experimental data on frequencies of vesicle movements in neurons $(8-40 \mathrm{~Hz})$ obtained by light scattering measurements [15].

Obviously, the cellular processes are likely to influence one another. Let us, therefore, investigate each frequency range in view of detecting such interactions. Figure 3 presents variations of the main frequency components with time. In the low frequency range $(0-5 \mathrm{~Hz})$ [Fig. 3(a)], four rhythmic components $(0.1,0.3,1$, and 2$4 \mathrm{~Hz}$ ) are stable during the whole time of observation. Rhythms between 0.1 and $0.3 \mathrm{~Hz}$ maintain constant values in time while rhythms between 1 and $2-4 \mathrm{~Hz}$ demonstrate variations caused by slower components. In the high frequency range $(10-20 \mathrm{~Hz})$, we find a large number of coexisting rhythmic components with quite nonstationary behavior and different modulation properties [Fig. 3(b)].

Modulation of fast oscillations by a slower dynamics can be considered as a form of nonlinear interaction between specified modes, and this phenomenon may help to identify observed frequencies with particular cellular processes. To obtain an overview of the dynamical properties of cellular regulation, we analyzed about 200 recordings to get a distribution over the depth and frequency of modulation. For each recording, the double-wavelet analysis reveals the rhythmic components involved in the modulation process of the specific activity. In this way, a form of distribution is determined by the number of slower components participating in the modulation process and by the deviations over
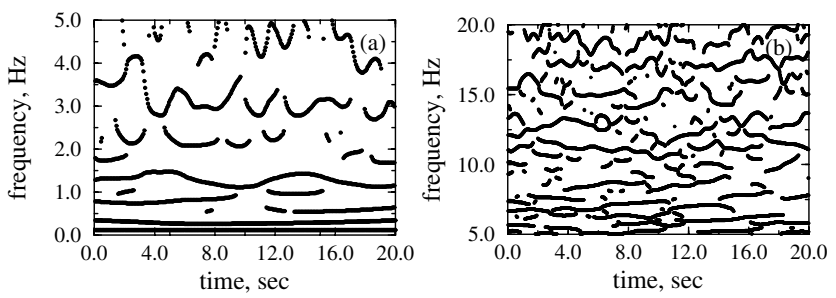

FIG. 3. Typical dynamics of all local maxima of the energy density for (a) the low and (b) high frequency ranges reveals variations of rhythmic behavior in time. recordings. Figures 4(a) and 4(b) illustrate that for the low frequency range, the rhythmic components at $1 \mathrm{~Hz}$ (black circles) and 2-4 Hz (white circles) are clearly separated with respect to depth of modulation in the case of frequency modulation [Fig. 4(a)] and with respect to modulation frequency in the case of amplitude modulation [Fig. 4(b)]. Moreover, as shown in Fig. 4(c), there is one well-defined peak in the spectrum of the modulation process. Such a well-defined separation can indicate different biological regulatory mechanisms of the 1 and $2-4 \mathrm{~Hz}$ rhythmic activities. We suggest that change of the neuron membrane potential under the rest condition has several components ( 1 and $2-4 \mathrm{~Hz}$ rhythms) that are modulated in different ways. The supposed changes of the transmembrane potential with $1 \mathrm{~Hz}$ frequency are influenced by constant $0.1 \mathrm{~Hz}$ process (lipid rafts and protein movements and redistribution of the membrane-bound ions being socalled intrinsic membrane properties). This assumption is in accordance with data on regulation of the ion channel activity and transmembrane potential by membrane $\mathrm{Ca}^{2+}$ and the formation of lipid clusters [16,17]. The change of the membrane potential with $2-4 \mathrm{~Hz}$ frequency is regulated by both intrinsic membrane properties and ion channel activity and, thus, is modulated by the 0.1 and $0.3 \mathrm{~Hz}$ rhythms.

In the high frequency range [Figs. 5(a) and 5(b)], the distribution of modulation frequencies for the considered rhythmic behaviors at 11 and $17 \mathrm{~Hz}$ is broader since more rhythmic components from the lower frequency range are involved in the modulation process [Fig. 5(c)]. Note that in spite of different contributions of the various rhythmic components, the structure of the modulation spectrum looks similar for the two components from the high frequency band. In this case, the absence of a clear distinction between the modulation rhythms allows us to assume that they originate from similar processes and a common biological regulation. Modulation of the 11 and $17 \mathrm{~Hz}$ rhythms by $0.1,0.3$, and $0.8-1 \mathrm{~Hz}$ components supports our suggestion about their origin as spontaneous rhythmic activities of neurons, activities that are known to be regulated by plasma membrane properties, ion channel activity, and low frequency changes of the transmembrane potential [16]. Spontaneous rhythmic activity of the neuron depends on the electrical membrane properties and thus its frequen-

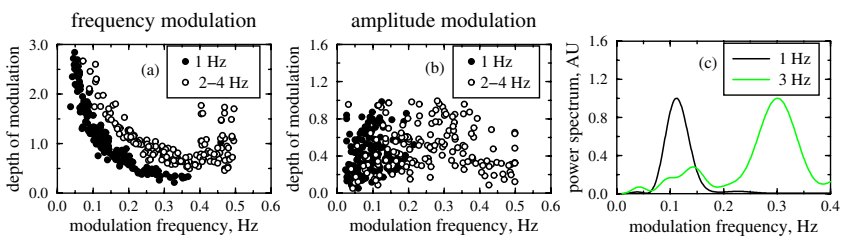

FIG. 4 (color online). Distribution of depth and modulation frequency for 1 and $2-4 \mathrm{~Hz}$ rhythmic components in the cases of (a) frequency and (b) amplitude modulation. (c) Typical normalized spectrum of the modulation process. 


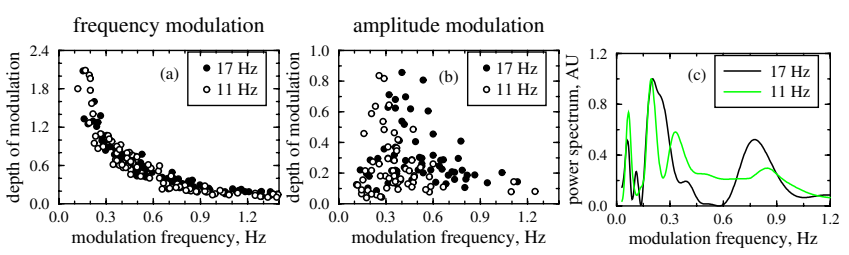

FIG. 5 (color online). Distribution of depth and modulation frequency for 11 and $17 \mathrm{~Hz}$ rhythmic components in the cases of (a) frequency and (b) amplitude modulation. (c) Typical normalized spectrum of the modulation process.

cies are modulated by processes that affect membrane potential, i.e., intrinsic membrane properties $(0.1 \mathrm{~Hz})$, the activity of ion channels (e.g., $\left.\mathrm{Ca}^{2+}\right)(0.3 \mathrm{~Hz})$, and subthreshold oscillations of the membrane potential $(0.8-1 \mathrm{~Hz})$. Movements of vesicles, particularly synaptic vesicles, depends on the concentration of intracellular $\mathrm{Ca}^{2+}$ and thus are also related to the membrane potential and to the activity of $\mathrm{Ca}_{V}^{2+}$ channels. Therefore, frequency changes of the vesicle movements in the cytoplasm can also be modulated by low frequency rhythms such as 0.3 and $1 \mathrm{~Hz}$. We suppose that frequencies in the low and high ranges can be partly related to the cytoskeleton reorganizations that occur under the function of the ion channels and organelles movements [18]. Recently it was shown that cytoskeleton in its turn regulates ion channel activity and location of mitochondria and endoplasmic reticulum and $\mathrm{Ca}^{2+}$ signaling between them $[19,20]$. Further studies of neuron function by means of interference microscopy and doublewavelet analysis may help to reveal the relation of the cytoskeleton organization to the activity of the plasma membrane and cytoplasmic processes.

In this Letter, interference microscopy recordings were examined by means of a double-wavelet analysis to explore the dynamical properties of intraneuron regulatory mechanisms operating at different time scales. We showed that at the rest conditions, neurons display a complex intrinsic optical activity. This is expressed through the changes of the cellular refractive index that are caused by various intracellular processes. The Letter for the first time established the characteristic frequencies of the refractive index changes in the range $0-25 \mathrm{~Hz}$ and revealed the presence of nonlinear interactions in the form of frequency and amplitude modulation of the fast oscillations by the slower processes. Further analysis of refractive index dynamics and relations between the revealed frequencies of the refractive index changes and identified cellular processes will be useful for better understanding of the function and interaction of different time-scale processes in neuron compartments at rest conditions as well as under the influence of external stimuli.

This work was supported by The European Commission (BioSim, Project No. 005137) and by the Danish National
Science Foundation. O.S. acknowledges support from Lundbeck Foundation. A. P. acknowledges support from the Russian Ministry of Education and Science and CRDF Y1-P-06-06. A. B. acknowledges support from Government of Russian Federation. We also acknowledge the company "Amphora Laboratories" (http://www.amphoralabs.ru) for providing the modulation interference microscope MIM-2.1 and for excellent technical assistance during the experiments.

[1] V. A. Andreev and K. V. Indukaev, J. Russ. Laser Res. 24, 220 (2003)

[2] V. P. Tychinskii, Physics Usp. 44, 617 (2001).

[3] T. A. Brindikova, S. M. Novikov, G. V. Maksimov, T. V. Vyshenskaya, and V.P. Tychinskiy, Eur. Biophys. J. 29, 351 (2000).

[4] G. V. Maksimov, S. L. Nikandrov, E. S. Lazareva, V.P. Tychinskii, and A.B. Rubin, Bulletin of experimental biology and medicine Biulleten eksperimentalnoi biologii i meditsiny 131, 457 (2001).

[5] V. A. Straub and P. R. Benjamin, J. Neurosci. 21, 1767 (2001).

[6] A. Grossmann and J. Morlet, SIAM J. Math. Anal. 15, 723 (1984); I. Daubechies, Ten Lectures on Wavelets (S.I.A.M., Philadelphia, 1992).

[7] G. Kaiser, A Friendly Guide to Wavelets (Birkhäuser, Boston, 1994).

[8] O. V. Sosnovtseva, A. N. Pavlov, E. Mosekilde, N.-H. Holstein-Rathlou, and D. J. Marsh, Phys. Rev. E 70, 031915 (2004).

[9] M. Haller, S.L. Mironov, and D.W. Richter, J. Neurophysiol. 86, 412 (2001).

[10] R. A. Stepnoski, A. LaPorta, F. Raccuia-Behling, G. E. Blonder, R. E. Slusher, and D. Kleinfeld, Proc. Natl. Acad. Sci. U.S.A. 88, 9382 (1991).

[11] A. Szucs, G. Molnar, and K. S-Rozsa, Acta Biologica Academiae Scientiarum Hungaricae 50, 269 (1999).

[12] A. Schutt, T.H. Bullock, and E. Basar, Brain Research 879, 73 (2000).

[13] N. Peixoto and F. J. Ramirez, European Journal of Neuroscience 12, 155 (2000).

[14] A. Reboreda, E. Sanchez, M. Romero, and J. A. Lamas, J. Physiol. 551, 191 (2003).

[15] D. Landowne and L. B. Cohen, Biol. Bull. 137, 407 (1969).

[16] B. Hille, Ion Channels of Exitable Membranes (University of Washington, Washington, 1992).

[17] K. M. S. O'Connel, J.R. Martens, and M. M. Tamkun, Trends in cardiovascular medicine 14, 37 (2004).

[18] J. Metuzals, V. Montpetit, and D. F. Clapin, Cell Tissue Res. 214, 455 (1981).

[19] D. Hattan, E. Nesti, T. G. Cachero, and A. D. Morielli, J. Biol. Chem. 277, 38596 (2002).

[20] S. L. Mironov, M. V. Ivannikov, and M. Johansson, J. Biol. Chem. 280, 715 (2005). 